\title{
Classifying Complex Faraday Spectra with Convolutional Neural Networks
}

\author{
Shea Brown ${ }^{1 \star}$, Brandon Bergerud ${ }^{1}$, Allison Costa $^{1}$, B. M. Gaensler ${ }^{2}$, \\ Jacob Isbell ${ }^{1}$, Daniel LaRocca ${ }^{1}$, Ray Norris ${ }^{3}$, Cormac Purcell ${ }^{4}$, \\ Lawrence Rudnick ${ }^{5}$, Xiaohui Sun ${ }^{6}$ \\ ${ }^{1}$ Department of Physics \& Astronomy, The University of Iowa, Iowa City, IA, 52245 \\ ${ }^{2}$ Dunlap Institute for Astronomy and Astrophysics, The University of Toronto, Toronto, ON M5S 3H4, Canada \\ ${ }^{3}$ Western Sydney University, Locked Bag 1797, 1797, Penrith South, NSW, Australia \\ ${ }^{4}$ Research Centre for Astronomy, Astrophysics, and Astrophotonics, Macquarie University, NSW 2109, Australia \\ ${ }^{5}$ Minnesota Institute for Astrophysics, University of Minnesota, 116 Church Street SE, Minneapolis, MN 55455, USA \\ ${ }^{6}$ Department of Astronomy, Yunnan University, and Key Laboratory of Astroparticle Physics of Yunnan Province, Kunming, 650091, China
}

\begin{abstract}
Advances in radio spectro-polarimetry offer the possibility to disentangle complex regions where relativistic and thermal plasmas mix in the interstellar and intergalactic media. Recent work has shown that apparently simple Faraday Rotation Measure (RM) spectra can be generated by complex sources. This is true even when the distribution of RMs in the complex source greatly exceeds the errors associated with a single component fit to the peak of the Faraday spectrum. We present a convolutional neural network $(\mathrm{CNN})$ that can differentiate between simple Faraday thin spectra and those that contain multiple or Faraday thick sources. We demonstrate that this CNN, trained for the upcoming Polarisation Sky Survey of the Universe's Magnetism (POSSUM) early science observations, can identify two component sources $99 \%$ of the time, provided that the sources are separated in Faraday depth by $>10 \%$ of the FWHM of the Faraday Point Spread Function, the polarized flux ratio of the sources is $>0.1$, and that the Signal-to-Noise radio $(\mathrm{S} / \mathrm{N})$ of the primary component is $>5$. With this $\mathrm{S} / \mathrm{N}$ cut-off, the false positive rate (simple sources mis-classified as complex) is $<0.3 \%$. Work is ongoing to include Faraday thick sources in the training and testing of the CNN.
\end{abstract}

Key words: polarization - methods: data analysis - methods: statistical - methods: analytical - methods: numerical

\section{INTRODUCTION}

\subsection{Background}

Faraday rotation of linearly polarized radio emission gives unique insight into the properties of the intervening magneto-ionic medium. Measurements of rotation-measures of background polarized radio sources probes astrophysical magnetic fields in a variety of environments like the Solar corona (Kooi et al. 2017), H II regions (Harvey-Smith et al. 2011), the interstellar medium of the Milky Way (Han et al. 1997; Sun \& Reich 2010; Wolleben et al. 2010; Pshirkov et al. 2011; Van Eck et al. 2011; Jansson \& Farrar 2012; Akahori et al. 2013), external galaxies (Han et al. 1998; Gaensler et al. 2005; Mao et al. 2012; Bernet et al. 2013), and the intracluster (Bonafede et al. 2010, 2013) and intergalactic (Akahori

* E-mail:shea-brown@uiowa.edu
\& Ryu 2011; Akahori et al. 2014) medium. Traditionally, Faraday rotation has been measured by fitting the change in polarization angle $\chi$ as a function of wavelength squared $\left(\lambda^{2}\right)$, parameterized by the rotation measure (RM) defined by

$\chi\left(\lambda^{2}\right)=\chi_{0}+R M \lambda^{2}$,

where $\chi_{0}$ is the intrinsic polarisation angle of the radio emission. This linearity with $\lambda^{2}$ is only valid for the case of a single synchrotron emitting source with an intervening cloud of magnetised thermal plasma. The wide-band capability of modern radio telescopes has allowed the use of RM synthesis (Brentjens \& de Bruyn 2005; Sun et al. 2015), which can address problems of bandwidth depolarization and multiple emitting/rotating regions along the line of sight (or within the same beam). RM Synthesis inverts the complex polar- 
isation spectrum $P\left(\lambda^{2}\right)=Q\left(\lambda^{2}\right)+i U\left(\lambda^{2}\right)$ into a Faraday spectrum

$F(\phi)=K \int_{-\infty}^{+\infty} P\left(\lambda^{2}\right) \mathrm{e}^{-2 \mathrm{i} \phi\left(\lambda^{2}-\lambda_{0}^{2}\right)} \mathrm{d} \lambda^{2}$

where $K$ is a constant, and $\phi$ is the "Faraday depth" of the emission, given by

$\phi(r)=K^{\prime} \int_{\vec{r}}^{0} n_{e} \vec{B} \cdot \overrightarrow{\mathrm{d} l}$,

where $K^{\prime}$ is a constant, $n_{e}$ is the electron density, $\vec{B}$ is the magnetic field vector, and $\overrightarrow{\mathrm{d} l}$ is a infinitesimal distance along the line of sight from the synchrotron source located at $\vec{r}$. In the simple case described in Equation 1, $\phi=\mathrm{RM}$. The development of deconvolution algorithms for the Faraday spectra (Heald 2009) has further improved the ability of RM Synthesis to reveal multiple sources along the line of sight.

Farnsworth et al. (2011) described an ambiguity in RM derived from $\chi\left(\lambda^{2}\right)$ fitting and RM Synthesis where two Faraday component model can produce a consistent single component solution that is neither of the input components nor their mean. This ambiguity can lead to an error in $\phi$ derived from these methods that is greater than what one would naively calculate from the uncertainty in fitting the peak of $F(\phi)$. In the era of large radio surveys meant to produce grids of background RMs for the archival science, there is a need to distinguish between "simple" foreground screens and more complex sources.

One such survey is the Polarisation Sky Survey of the Universe's Magnetism (POSSUM, Gaensler et al. 2010), which will be conducted with the Australian Square Kilometre Array Pathfinder (ASKAP, Johnston et al. 2008) and will measure more than 1 million polarized sources in the frequency range of $1130-1430 \mathrm{MHz}$ over $75 \%$ of the sky. An Early Science survey, which is being conducted as part of ASKAP's science commissioning observations, will make use of only 12 antennae of ASKAP, but the frequency coverage will be extended to 700-1800 MHz. The extended frequency coverage of the Early Science survey is ideal for identifying and investigating complex Faraday spectra, provided that these spectra can be identified in an automated way. There are a variety of ways in which a Faraday spectrum can deviate from a single source with a foreground Faraday screen (called Faraday thin, Brentjens \& de Bruyn 2005). It can be Faraday thick (caused by significant mixing of Faraday rotating and emitting plasma), have multiple Faraday thin components, or there can be external/internal Faraday dispersion (modulation due to rapidly changing Faraday rotating cells along the line of sight, or within a single beam).

The initial data release of the POSSUM survey will be a catalogue of sources with simple Faraday spectra and their associated properties. Simple spectra come from sources with a single Faraday rotating screen in front of them, i.e., their polarisation angle would obey Equation 1 for all $\lambda^{2}$ values. This POSSUM Polarization Catalogue (PPC) will not include complex sources, which are any sources that are not simple as defined above, so the pipeline producing the catalogue must have an efficient and effective way of determining/testing the complexity of a source. This test needs to be able to: 1) determine whether a Faraday spectrum is complex in general, including cases where there are more than one well separated Faraday sources (peaks) in the spectrum, 2) determine whether a given peak is Faraday thin or not, and 3) provide some way of assessing how sure we are of the resulting classification. Initial work has shown that the second moment of the clean components resulting from a Faraday cleaning procedure can provide some discriminating power (e.g., Brown 2011; Anderson et al. 2015), while more recent work has focused on the model fitting the polarization spectrum and examining the statistically more likely model in a Bayesian sense (e.g., O'Sullivan et al. 2012; Sun et al. 2015; O'Sullivan et al. 2017; Purcell \& West 2017).

To this end, we present the construction of a convolutional neural network (CNN) that can classify a Faraday spectrum as either simple or complex. In $\S 2$ we describe the construction and training of the $\mathrm{CNN}$, in $\S 3$ we outline the testing of the network on simulated data, and in $\S 4$ we summarize the limitations of the metric and discuss future improvements.

\section{CONVOLUTIONAL NEURAL NETWORK}

A few of the major difficulties for developing a test for complexity are A) the ad hoc nature of the initial choice of a metric, B) the significant work required to find a metric threshold appropriate for the science goals, and C) estimating the uncertainty in the accuracy of the metric/threshold combination. For these reasons, we have explored convolutional neural networks (CNNs, Le Cun \& Bengio 1995; Krizhevsky, Sutskever \& Hinton 2012), in particular the "inception" networks developed by the GoogLeNet team (Szegedy et al. 2014) as potential classifiers. CNNs are ideal for this problem, as they apply a series of convolutions to the data, along with non-linear intermediate functions (e.g., rectified linear units, or ReLUs, Nair \& Hinton 2010), and the training process will find the series of convolutional kernels that are optimal for classifying the spectra. While model fitting requires large searches over parameter space each time a spectrum is analyzed, a CNN samples the space only during training, and requires only a straightforward and efficient feedforward network of matrix operations to classify the sources. In the inception model a series of convolutions is done in parallel, each with a different kernel size, further allowing the network to search for features in the data that distinguish complex sources. In essence, the network will find the best metric to use during the training process, eliminating the need to choose one at the outset.

We considered and tested several convolutional neural networks, and the current best network has three convolutional inception layers, along with two fully-connected (dense) layers. Each inception layer has three parallel channels of convolutions, with kernel sizes of 3,5, and 23 channels (in Faraday space each channel is $1 \mathrm{rad} / \mathrm{m}^{2}$ ), as well as two channels with a " $1 \times 1$ " convolutions, one of which also has a maxpooling layer (Boureau et al. 2010). The 1x1 convolution essentially allows mixing between the real and imaginary parts of the spectrum in the first application, and serve to reduce the number of parameters in the two subsequent layers (Szegedy et al. 2014). The full network is show in Fig. 1, and the individual inception layers are shown in Fig. 2. The "Flatten" layer will take the deep network of features constructed by the inception layers and project it into a vector 


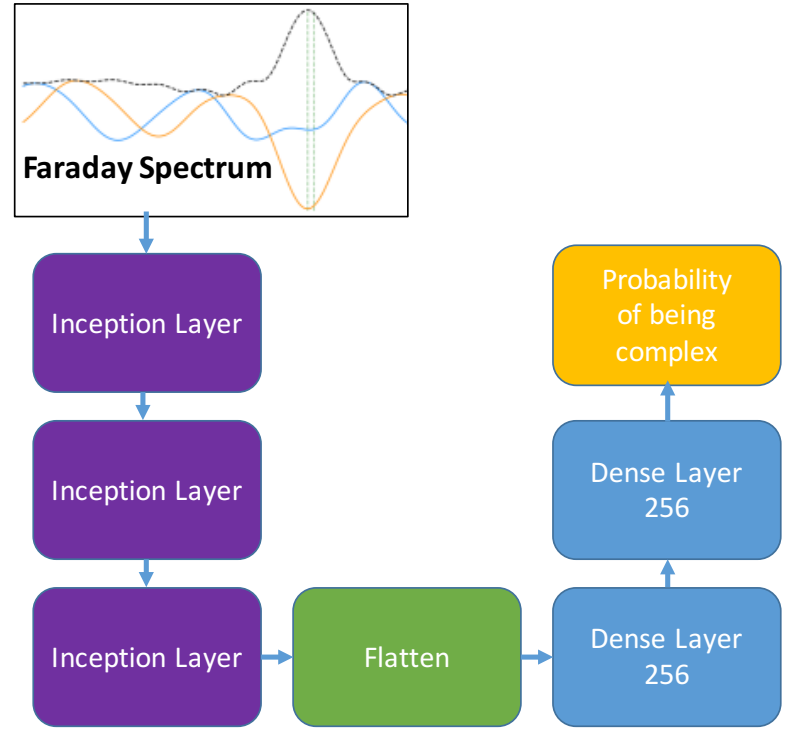

Figure 1. Three inception layer convolutional neural network (CNN) classifier. Each dense layer has an additional dropout (0.5) and activation (ReLU) layer within it.

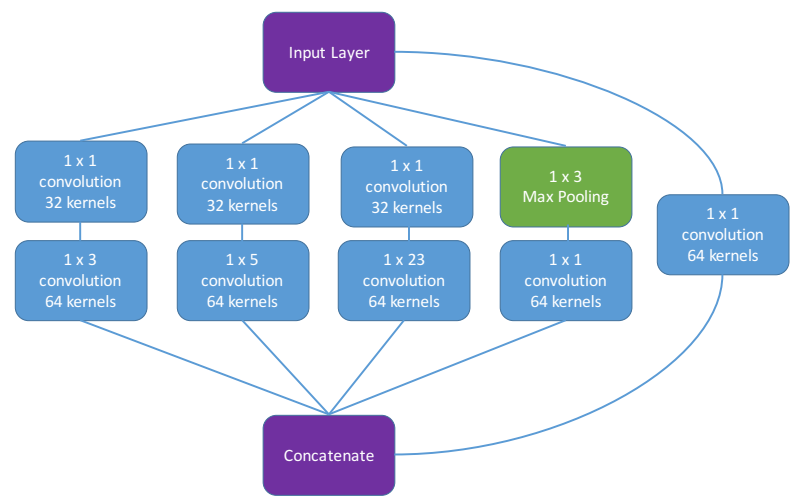

Figure 2. A zoom in of an inception layer. Each of the convolutional layers (in blue) has additional batch-normalization and activation (ReLU) layers within it.

of features to be passed to the dense layers. These dense layers are traditional artificial neural networks (Cybenko 1989). Source code can be found on Github ${ }^{1}$.

\section{TRAINING}

In order to train the proposed complexity classifier, we simulated both simple and complex spectra using a realistic observational model. Since our current purpose is to develop a metric for the POSSUM Early Science observations, we have used the proposed frequency coverage of the ASKAP 12 Early Science survey (700-1300 MHz, 1500-1800 MHz),

\footnotetext{
1 https://github.com/sheabrown/faraday_complexity/blob/ master/final/How2Guide.ipynb
}

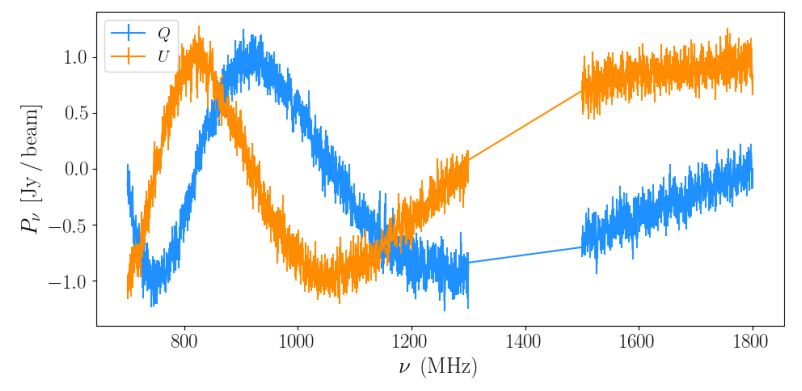

Figure 3. An example of a polarized spectrum sampled with the POSSUM Early Science frequency coverage used in the training data.

with a total of 900, $1 \mathrm{MHz}$ channels (see Fig. 3). The current work focuses only on two-component models (with both components being Faraday thin), which are believed to be the dominant source of complex spectra (e.g., Anderson et al. 2015; O'Sullivan et al. 2017). The two emitting regions can have different polarized amplitudes $\left(P_{1}\right.$ and $\left.P_{2}\right)$, as well as different intrinsic polarization angles $\left(\chi_{1}\right.$ and $\left.\chi_{2}\right)$ and foreground Faraday depths $\left(\phi_{1}\right.$ and $\left.\phi_{2}\right)$. The combined polarized spectrum is given by

$P\left(\lambda^{2}\right)=P_{1} e^{\left[2 i\left(\chi_{1}+\phi_{1} \lambda^{2}\right)\right]}+P_{2} e^{\left[2 i\left(\chi_{2}+\phi_{2} \lambda^{2}\right)\right]}$.

We also simulate simple sources using Equation 4, but with $P_{2}=0$. To train the network, we generated 130,000 sources (100,000 training set and 30,000 for validation), roughly half of which were complex (two-component) sources, and the other half were simple ${ }^{2}$. Table 1 shows the parameter space that was sampled at random from a uniform distribution. We used the simplifying assumption that both the noise and total intensity have no spectral dependence. As a first step, we chose to train the network on the Faraday spectra $F(\phi)$ only, as it will be computed as part of the POSSUM pipeline, but in theory the network can be trained using the polarization spectrum directly as well. For each source, a polarization spectrum was created first, and then a Faraday spectrum was created using the standard inversion formula of Brentjens \& de Bruyn (2005),

$F(\phi) \approx K \sum_{i=1}^{N} P_{i} \mathrm{e}^{-2 \mathrm{i} \phi\left(\lambda_{i}^{2}-\lambda_{0}^{2}\right)}$,

where $K=1 / N_{\text {channels }}, \lambda_{0}^{2}$ is the average $\lambda^{2}$ of the channels, and $P_{i}$ is the measured complex polarization in channel $i$. Figure 3 shows an example of a complex polarized $P(\nu)$, and Fig. 4 shows a selection of Faraday $F(\phi)$ spectra for complex sources in the training set.

The network was trained using batch stochastic gradient-descent (Duda et al. 2012) on the training set of 100,000 sources, with 30,000 sources withheld for crossvalidation during the training. The training lasted for 100

2 Each time the data simulator created a spectrum, there was a $50 \%$ probability that it would be complex. The probability is an adjustable parameter in the source code. 
Table 1. Two-component Parameter Space

\begin{tabular}{lcc}
\hline Parameter & Symbol & Range \\
\hline \hline Amplitude 1 & $P_{1}$ & 1 \\
Amplitude 2 & $P_{2}$ & {$[0,1]$} \\
Faraday depth $\{1,2\}$ & $\phi_{\{1,2\}}$ & {$[-50,+50]$} \\
Polarization angle $\{1,2\}$ & $\chi_{\{1,2\}}$ & {$[0,+\pi]$} \\
Noise/Channel & $\sigma$ & {$[0,0.333]$} \\
\hline \hline
\end{tabular}

epochs, though no improvement on the validation set was found after 55 epochs. The weights found on epoch 55 were saved and used for testing.

\section{RESULTS \& DISCUSSION}

The trained network was then applied to 100,000 new sources randomly generated using the same parameter space as the training set. The output for each source is a value $p$ between 0 and 1 which can be thought of as the probability that the source is complex. Figure 5 shows a histogram of $p$ for the 100,000 test sources. The distribution is bi-modal, indicating that the network was confident about the classification of most sources. If we take $p>0.5$ as the threshold for complexity, we can construct the confusion matrix as shown in Table 2.

The network produces $7.2 \%$ false negatives and $3.0 \%$ false positives. In order to hunt down the complex sources that are mis-classified as simple, we can plot the $p$ for all the complex sources as a function of both the second component's amplitude $P_{2}$ and the absolute separation in the two components' Faraday depths $\Delta \phi=\left|\phi_{1}-\phi_{2}\right|$ (Fig. 6). The majority of false negatives happen at small $P_{2}$ and $\Delta \phi$. This is consistent with the results of Farnsworth et al. (2011) and Sun et al. (2015) that point to the difficulty in identifying two component sources when $\Delta \phi<F W H M$ of the Faraday Point Spread Function. Figure 7 shows an example of one of the false-negatives.

For the purposes of constructing a classifier for largescale polarsation surveys like POSSUM, we would like to exclude the phase space of sources that would likely not make it into the catalog due to low signal-to-noise, as well as sources where the rotation measures of the two components are close enough to allow probing of a foreground Faraday screen. We therefore searched for the region of phase space in which we can detect $>99 \%$ of the complex sources, allowing for the false positive rate to adjust appropriately based on the cut-off values. We found that if the minimum signalto-noise of the primary component is 5.0, and restrict our sample to $P_{2}>0.1$, and $\Delta \phi>2.3 \mathrm{rad} / \mathrm{m}^{2}$ (which is about $10 \%$ of the $23 \mathrm{rad} / \mathrm{m}^{2}$ FWHM of the Faraday point spread function), the false negatives are reduced to $<1 \%$, while the false positives rate reduces to $<0.3 \%$. Table 3 shows the new confusion matrix with the cut-offs applied to the same simulated data. What these cutoffs mean for the initial POSSUM catalog (the PPC) is that the network is $99 \%$ confident that the Faraday spectrum is simple, with the understanding that a secondary component can be hiding in the above phase space. The probability returned by the network can be recorded for each source, allowing one to flag sources where
Table 2. Confusion Matrix: Before Cutoffs

\begin{tabular}{lcc}
\hline Predicted $->$ & Simple & Complex \\
\hline \hline True Simple & 48,318 & 1481 \\
True Complex & 3,618 & 46,583 \\
\hline
\end{tabular}

Table 3. Confusion Matrix: After Cutoffs

\begin{tabular}{lcc}
\hline Predicted $->$ & Simple & Complex \\
\hline \hline True Simple & 29,281 & 69 \\
True Complex & 247 & 25,337 \\
\hline
\end{tabular}

$p$ is close to the nominal cutoff of $p<0.5$ for the PPC. We should note that one can trade a higher $\mathrm{S} / \mathrm{N}$ cutoff to allow a narrower $\Delta \phi$ and still reach the $99 \%$, something that might be advantageous depending on the science goal.

\subsection{Conclusion \& Future Work}

We have constructed a convolutional neural network that is able to distinguish between simple Faraday sources and those that contain two Faraday thin components, demonstrating on simulated POSSUM Early Science data that it can detect $99 \%$ of complex sources with $<0.3 \%$ false positive rate in a realistic and useful region of the source parameter phase-space. The training and application of this network for other observational parameters in straightforward, needing only the frequency coverage to be changed. The most obvious future development of the network would include 1) lifting the simplification on the flat spectral index and channel independent noise, 2) allowing for modified RM Synthesis that includes channel weights in Equation 2, and 3) the inclusion of Faraday thick and three component sources during training. Including complexity beyond this may prove impractical, as O'Sullivan et al. (2017) was able to fit just about any source using a combination of three Faraday thin components. Given the power of CNNs used in commercial applications, the inclusion of 3) in to the training would also allow for multiple classes beyond a binary simple/complex classification.

The Dunlap Institute is funded through an endowment established by the David Dunlap family and the University of Toronto. B.M.G. acknowledges the support of the Natural Sciences and Engineering Research Council of Canada (NSERC) through grant RGPIN-2015-05948, and of the Canada Research Chairs program. Partial support for LR comes from NSF grant 17-14205 to the University of Minnesota. XHS is supported by the National Natural Science Foundation of China under grant No. 11763008.

\section{REFERENCES}

Akahori, T., \& Ryu, D. 2011, ApJ, 738, 134

Akahori, T., Ryu, D., Kim, J., \& Gaensler, B. M. 2013, ApJ, 767, 150

Akahori, T., Kumazaki, K., Takahashi, K., \& Ryu, D. 2014, PASJ, 66,65 
Complex Sources
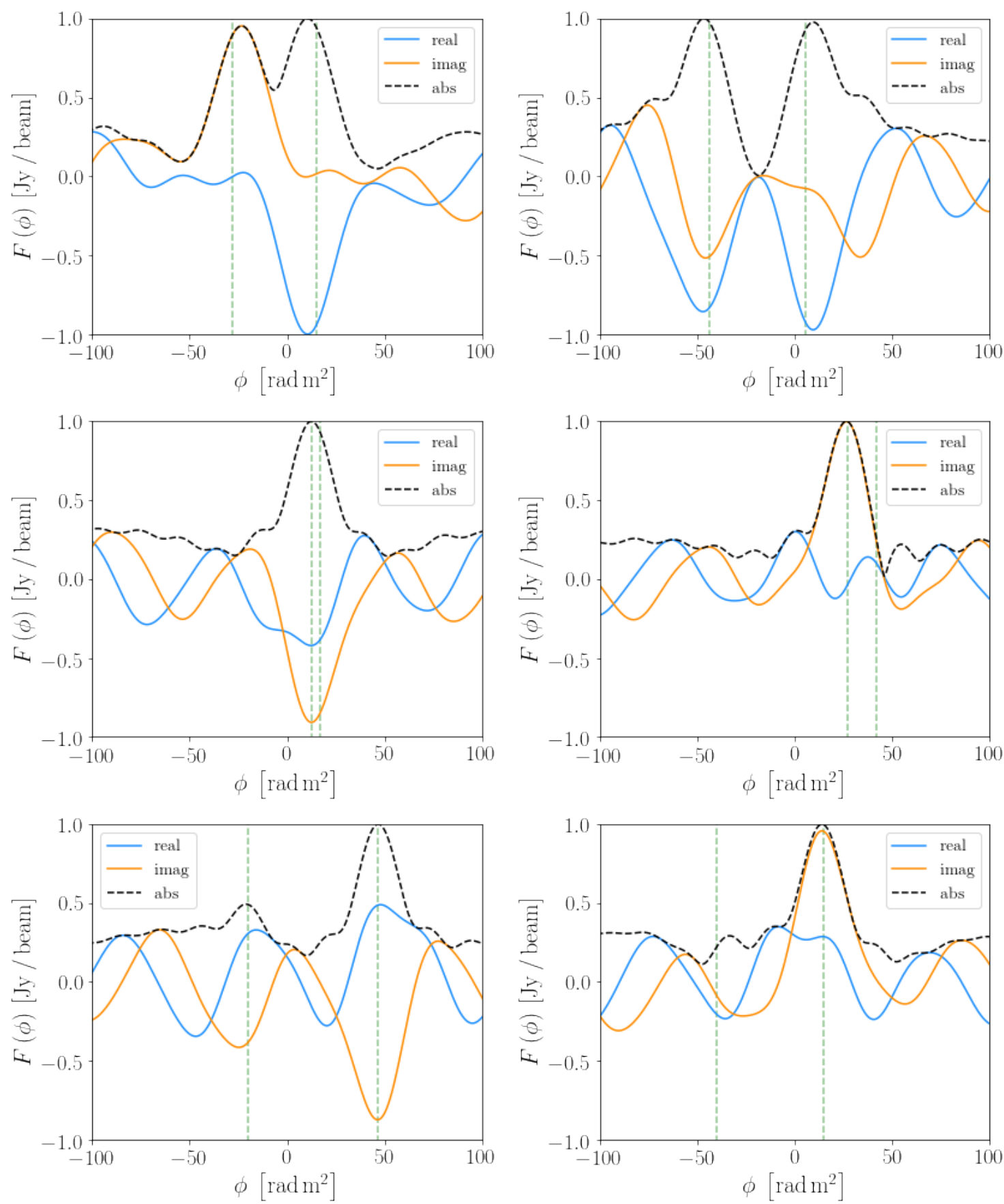

Figure 4. Complex Faraday spectra from the training data (with $\phi_{1}$ and $\phi_{2}$ given by the vertical green dashed lines). No deconvolution (RM Clean) was performed.

Anderson, C. S., Gaensler, B. M., Feain, I. J., \& Franzen, T. M. O. 2015, ApJ, 815, 49

Andrecut, M. 2013, MNRAS, 430, L15

Aniyan, A. K., \& Thorat, K. 2017, ApJS, 230, 20

Beck, R., Frick, P., Stepanov, R., \& Sokoloff, D. 2012, A\&A, 543, A113

Bell, M. R., Oppermann, N., Crai, A., \& Enßlin, T. A. 2013, A\&A, 551, L7
Bernet, M. L., Miniati, F., \& Lilly, S. J. 2013, ApJ, 772, L28

Bonafede, A., Feretti, L., Murgia, M., et al. 2010, A\&A, 513, A30

Bonafede, A., Vazza, F., Brüggen, M., et al. 2013, MNRAS, 433, 3208

Boureau, Y.-L., Ponce, J., \& LeCun, Y. 2010, in Proceedings of the 27 th international conference on machine learning (ICML10), $111 ? 118$

Brentjens, M. A., \& de Bruyn, A. G. 2005, A\&A, 441, 1217 


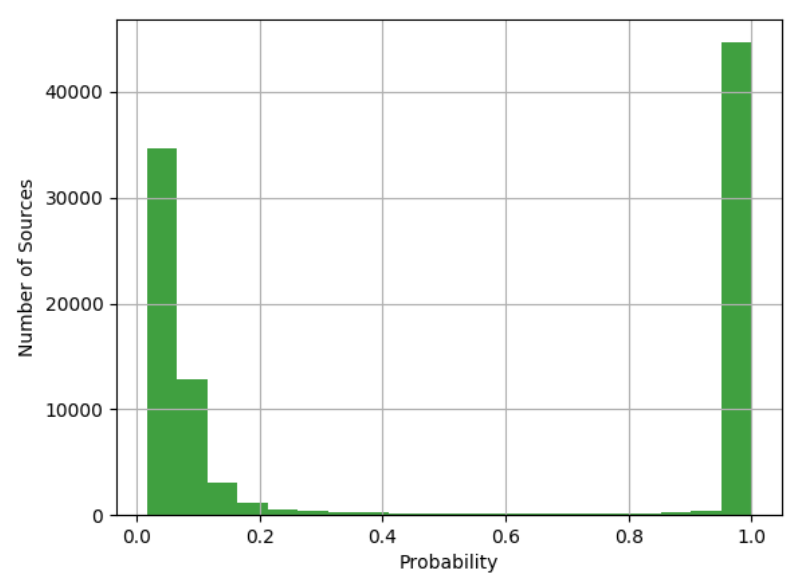

Figure 5. Histogram of probability values for the 100,000 test sources.

Brown, S. 2011, Internal POSSUM Report \#9: Assess Complexity of RM Synthesis Spectrum

Burn, B. J. 1966, MNRAS, 133, 67

Cybenko, G. 1989, Mathematics of control, signals and systems, 2,303

Duda, R. O., Hart, P. E., \& Stork, D. G. 2012, Pattern classification (John Wiley \& Sons)

Farnsworth, D., Rudnick, L., \& Brown, S. 2011, AJ, 141, 191

Gaensler, B. M., Haverkorn, M., Staveley-Smith, L., et al. 2005, Science, 307, 1610

Gaensler, B. M., Landecker, T. L., Taylor, A. R., \& POSSUM Collaboration 2010, Bulletin of the American Astronomical Society, 42, 470.13

Han, J. L., Manchester, R. N., Berkhuijsen, E. M., \& Beck, R. 1997, A\&A, 322, 98

Han, J. L., Beck, R., \& Berkhuijsen, E. M. 1998, A\&A, 335, 1117

Harvey-Smith, L., Madsen, G. J., \& Gaensler, B. M. 2011, ApJ, 736,83

Heald, G. 2009, Cosmic Magnetic Fields: From Planets, to Stars and Galaxies, 259, 591

Horellou, C., \& Fletcher, A. 2014, MNRAS, 441, 2049

Jansson, R., \& Farrar, G. R. 2012, ApJ, 757, 14

Johnston, S., Taylor, R., Bailes, M., et al. 2008, Experimental Astronomy, 22, 151

Kooi, J. E., Fischer, P. D., Buffo, J. J., \& Spangler, S. R. 2017, Sol. Phys., 292, 56

Krizhevsky, A., Sutskever, I., \& Hinton, G. E. 2012, in Advances in neural information processing systems, 1097?1105

Lamee, M., Rudnick, L., Farnes, J. S., et al. 2016, ApJ, 829, 5

LeCun, Y., \& Bengio, Y. 1995, The handbook of brain theory and neural networks, 3361, 1995

Li, F., Brown, S., Cornwell, T. J., \& de Hoog, F. 2011, A\&A, 531, A126

Mao, S. A., McClure-Griffiths, N. M., Gaensler, B. M., et al. 2012, ApJ, 759, 25

Nair, V., \& Hinton, G. E. 2010, in Proceedings of the 27th International Conference on Machine Learning (ICML-10), 807?814

O'Sullivan, S. P., Brown, S., Robishaw, T., et al. 2012, MNRAS, 421,3300

O'Sullivan, S. P., Purcell, C. R., Anderson, C. S., et al. 2017, MNRAS, 469, 4034

Pshirkov, M. S., Tinyakov, P. G., Kronberg, P. P., \& NewtonMcGee, K. J. 2011, ApJ, 738, 192

Purcell, C. R., \& West, J., 2017, Internal POSSUM Report \#68: Measuring Faraday Complexity
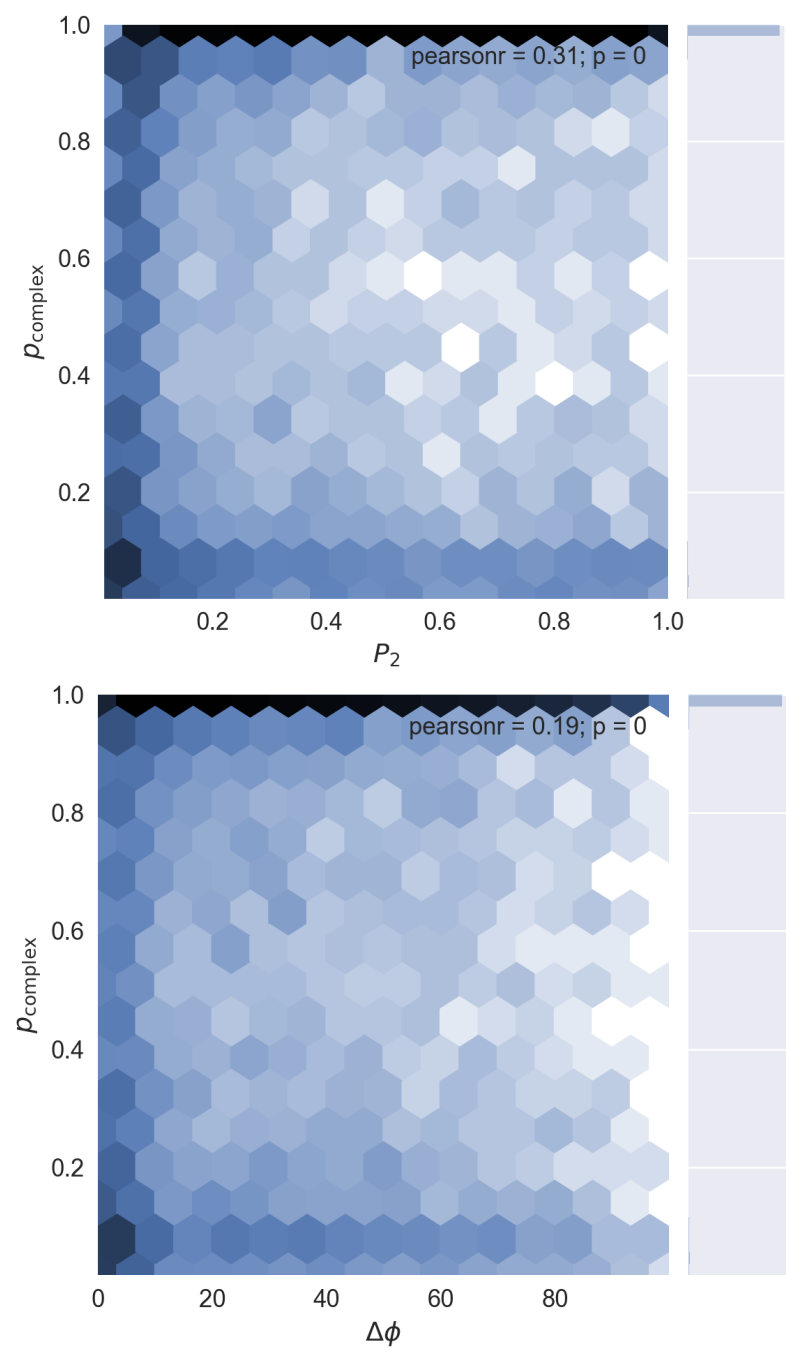

Figure 6. Top: Probability output of the network vs. the relative flux of the second component for simulated complex sources in the test data set. Colour scale is the log of the number of sources. The black triangles at the top are the high density of $p$ values around 0.99 , and the right sub-plot is a histogram showing that the vast majority of sources were classified as complex. Bottom: Like above, but plotted vs. the absolute difference in the Faraday depth $(\Delta \phi)$ between the two components.

Sun, X.-H., \& Reich, W. 2010, Research in Astronomy and Astrophysics, 10, 1287

Szegedy, C., Liu, W., Jia, Y., et al. 2014, arXiv:1409.4842

Schnitzeler, D. H. F. M., \& Lee, K. J. 2017, MNRAS, 466, 378

Sun, X. H., Rudnick, L., Akahori, T., et al. 2015, AJ, 149, 60

Vacca, V., Oppermann, N., Enßlin, T., et al. 2016, A\&A, 591, A13

Van Eck, C. L., Brown, J. C., Stil, J. M., et al. 2011, ApJ, 728, 97

Wolleben, M., Landecker, T. L., Hovey, G. J., et al. 2010, AJ, 139,1681

This paper has been typeset from a $\mathrm{T}_{\mathrm{E}} \mathrm{X} / \mathrm{L}_{\mathrm{A}} \mathrm{T}_{\mathrm{E}} \mathrm{X}$ file prepared by the author. 


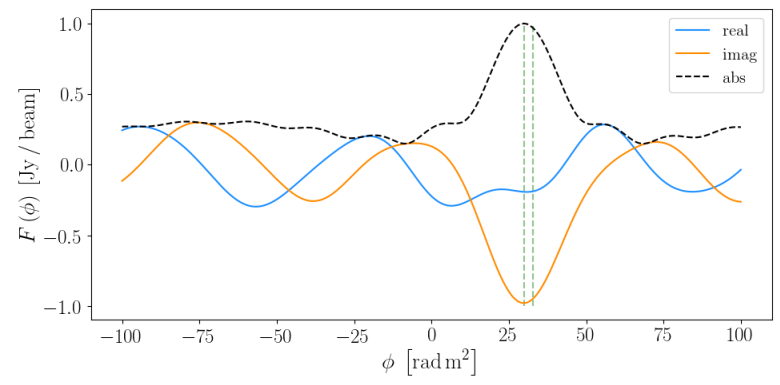

Figure 7. Top: Faraday spectrum of a complex source misidentified as simple by the classifier. The two Faraday depths are labeled with vertical green dashed lines. 